\title{
Basic Properties of the Rank of Matrices over a Field
}

\author{
Karol Pa̧k \\ Institute of Computer Science \\ University of Białystok \\ Poland
}

\begin{abstract}
Summary. In this paper I present selected properties of triangular matrices and basic properties of the rank of matrices over a field.

I define a submatrix as a matrix formed by selecting certain rows and columns from a bigger matrix. That is in my considerations, as an array, it is cut down to those entries constrained by row and column. Then I introduce the concept of the rank of a $m \times n$ matrix $A$ by the condition: $A$ has the rank $r$ if and only if, there is a $r \times r$ submatrix of $A$ with a non-zero determinant, and for every $k \times k$ submatrix of $A$ with a non-zero determinant we have $k \leq r$.

At the end, I prove that the rank defined by the size of the biggest submatrix with a non-zero determinant of a matrix $A$, is the same as the maximal number of linearly independent rows of $A$.
\end{abstract}

MML identifier: MATRIX13, version: 7.8.05 4.87.985

The articles [27], [10], [37], [23], [1], [2], [12], [38], [39], [7], [8], [3], [4], [24], $[36],[31],[15],[6],[13],[28],[14],[41],[30],[19],[34],[42],[9],[22],[16],[11]$, [25], [40], [18], [20], [26], [33], [21], [17], [35], [32], [29], [43], and [5] provide the terminology and notation for this paper.

\section{Triangular Matrices}

For simplicity, we use the following convention: $x, X, Y$ are sets, $D$ is a non empty set, $i, j, k, m, n, m^{\prime}, n^{\prime}$ are elements of $\mathbb{N}, i_{0}, j_{0}, n_{0}, m_{0}$ are non zero elements of $\mathbb{N}, K$ is a field, $a, b$ are elements of $K, p$ is a finite sequence of elements of $K$, and $M$ is a matrix over $K$ of dimension $n$.

Next we state a number of propositions: 
(1) For every matrix $A$ over $D$ of dimension $n \times m$ holds if $n=0$, then $m=0$ iff len $A=n$ and width $A=m$.

(2) The following statements are equivalent

(i) $\quad M$ is a lower triangular matrix over $K$ of dimension $n$,

(ii) $\quad M^{\mathrm{T}}$ is an upper triangular matrix over $K$ of dimension $n$.

(3) The diagonal of $M=$ the diagonal of $M^{\mathrm{T}}$.

(4) Let $p_{1}$ be an element of the permutations of $n$-element set. Suppose $p_{1} \neq \operatorname{idseq}(n)$. Then there exists $i$ such that $i \in \operatorname{Seg} n$ and $p_{1}(i)>i$ and there exists $j$ such that $j \in \operatorname{Seg} n$ and $p_{1}(j)<j$.

(5) Let $M$ be a matrix over $K$ of dimension $n$ and $p_{1}$ be an element of the permutations of $n$-element set. Suppose that

(i) $\quad p_{1} \neq \operatorname{idseq}(n)$, and

(ii) $\quad M$ is a lower triangular matrix over $K$ of dimension $n$ or an upper triangular matrix over $K$ of dimension $n$.

Then (the product on paths of $M)\left(p_{1}\right)=0_{K}$.

(6) Let $M$ be a matrix over $K$ of dimension $n$ and $I$ be an element of the permutations of $n$-element set. If $I=\operatorname{idseq}(n)$, then the diagonal of $M=I$-Path $M$.

(7) Let $M$ be an upper triangular matrix over $K$ of dimension $n$. Then Det $M=($ the multiplication of $K) \circledast($ the diagonal of $M)$.

(8) Let $M$ be a lower triangular matrix over $K$ of dimension $n$. Then Det $M=($ the multiplication of $K) \circledast($ the diagonal of $M)$.

(9) For every finite set $X$ and for every $n$ holds $\overline{\overline{\{Y ; Y \text { ranges over subsets of } X: \operatorname{card} Y=n\}}}=\left(\begin{array}{c}\operatorname{card} X \\ n\end{array}\right)$.

(10) $\overline{\overline{2 \operatorname{Set} \operatorname{Seg} n}}=\left(\begin{array}{l}n \\ 2\end{array}\right)$.

(11) Let $R$ be an element of the permutations of $n$-element set. If $R=$ $\operatorname{Rev}(\operatorname{idseq}(n))$, then $R$ is even iff $\left(\begin{array}{l}n \\ 2\end{array}\right) \bmod 2=0$.

(12) Let $M$ be a matrix over $K$ of dimension $n$ and $R$ be a permutation of $\operatorname{Seg} n$. Suppose $R=\operatorname{Rev}(\operatorname{idseq}(n))$ and for all $i, j$ such that $i \in \operatorname{Seg} n$ and $j \in \operatorname{Seg} n$ and $i+j \leq n$ holds $M_{i, j}=0_{K}$. Then $M \cdot R$ is an upper triangular matrix over $K$ of dimension $n$.

(13) Let $M$ be a matrix over $K$ of dimension $n$ and $R$ be a permutation of $\operatorname{Seg} n$. Suppose $R=\operatorname{Rev}(\operatorname{idseq}(n))$ and for all $i, j$ such that $i \in \operatorname{Seg} n$ and $j \in \operatorname{Seg} n$ and $i+j>n+1$ holds $M_{i, j}=0_{K}$. Then $M \cdot R$ is a lower triangular matrix over $K$ of dimension $n$.

(14) Let $M$ be a matrix over $K$ of dimension $n$ and $R$ be an element of the permutations of $n$-element set. Suppose that

(i) $\quad R=\operatorname{Rev}(\operatorname{idseq}(n))$, and

(ii) for all $i, j$ such that $i \in \operatorname{Seg} n$ and $j \in \operatorname{Seg} n$ and $i+j \leq n$ holds $M_{i, j}=0_{K}$ or for all $i, j$ such that $i \in \operatorname{Seg} n$ and $j \in \operatorname{Seg} n$ and $i+j>n+1$ 
holds $M_{i, j}=0_{K}$.

Then Det $M=(-1)^{\operatorname{sgn}(R)}$ (the multiplication of $K \odot(R$-Path $\left.M)\right)$.

(15) Let $M$ be a matrix over $K$ of dimension $n$ and $M_{1}, M_{2}$ be upper triangular matrices over $K$ of dimension $n$. Suppose $M=M_{1} \cdot M_{2}$. Then

(i) $\quad M$ is an upper triangular matrix over $K$ of dimension $n$, and

(ii) the diagonal of $M=\left(\right.$ the diagonal of $\left.M_{1}\right) \bullet\left(\right.$ the diagonal of $\left.M_{2}\right)$.

(16) Let $M$ be a matrix over $K$ of dimension $n$ and $M_{1}, M_{2}$ be lower triangular matrices over $K$ of dimension $n$. Suppose $M=M_{1} \cdot M_{2}$. Then

(i) $\quad M$ is a lower triangular matrix over $K$ of dimension $n$, and

(ii) the diagonal of $M=\left(\right.$ the diagonal of $\left.M_{1}\right) \bullet\left(\right.$ the diagonal of $\left.M_{2}\right)$.

\section{The Rank of Matrices}

Let $D$ be a non empty set, let $M$ be a matrix over $D$, let $n, m$ be natural numbers, let $n_{1}$ be an element of $\mathbb{N}^{n}$, and let $m_{1}$ be an element of $\mathbb{N}^{m}$. The functor $\operatorname{Segm}\left(M, n_{1}, m_{1}\right)$ yielding a matrix over $D$ of dimension $n \times m$ is defined as follows:

(Def. 1) For all natural numbers $i, j$ such that $\langle i, j\rangle \in$ the indices of $\operatorname{Segm}\left(M, n_{1}, m_{1}\right)$ holds $\left(\operatorname{Segm}\left(M, n_{1}, m_{1}\right)\right)_{i, j}=M_{n_{1}(i), m_{1}(j)}$.

For simplicity, we follow the rules: $A$ denotes a matrix over $D, A^{\prime}$ denotes a matrix over $D$ of dimension $n^{\prime} \times m^{\prime}, M^{\prime}$ denotes a matrix over $K$ of dimension $n^{\prime} \times m^{\prime}, n_{1}, n_{2}, n_{3}$ denote elements of $\mathbb{N}^{n}, m_{1}, m_{2}$ denote elements of $\mathbb{N}^{m}$, and $M$ denotes a matrix over $K$.

Next we state a number of propositions:

(17) If $\left[: \operatorname{rng} n_{1}, \operatorname{rng} m_{1}: \subseteq\right.$ the indices of $A$, then $\langle i, j\rangle \in$ the indices of $\operatorname{Segm}\left(A, n_{1}, m_{1}\right)$ iff $\left\langle n_{1}(i), m_{1}(j)\right\rangle \in$ the indices of $A$.

(18) If $\left[: \operatorname{rng} n_{1}, \operatorname{rng} m_{1}\right] \subseteq$ the indices of $A$ and $n=0$ iff $m=0$, then $\left(\operatorname{Segm}\left(A, n_{1}, m_{1}\right)\right)^{\mathrm{T}}=\operatorname{Segm}\left(A^{\mathrm{T}}, m_{1}, n_{1}\right)$.

(19) If $\left[: \operatorname{rng} n_{1}, \operatorname{rng} m_{1}: \subseteq\right.$ the indices of $A$ and if $m=0$, then $n=0$, then $\operatorname{Segm}\left(A, n_{1}, m_{1}\right)=\left(\operatorname{Segm}\left(A^{\mathrm{T}}, m_{1}, n_{1}\right)\right)^{\mathrm{T}}$.

(20) For every matrix $A$ over $D$ of dimension 1 holds $A=\left\langle\left\langle A_{1,1}\right\rangle\right\rangle$.

(21) If $n=1$ and $m=1$, then $\operatorname{Segm}\left(A, n_{1}, m_{1}\right)=\left\langle\left\langle A_{n_{1}(1), m_{1}(1)}\right\rangle\right\rangle$.

(22) For every matrix $A$ over $D$ of dimension 2 holds $A=\left(\begin{array}{ll}A_{1,1} & A_{1,2} \\ A_{2,1} & A_{2,2}\end{array}\right)$.

(23) If $n=2$ and $m=2$, then $\operatorname{Segm}\left(A, n_{1}, m_{1}\right)=$ $\left(\begin{array}{ll}A_{n_{1}(1), m_{1}(1)} & A_{n_{1}(1), m_{1}(2)} \\ A_{n_{1}(2), m_{1}(1)} & A_{n_{1}(2), m_{1}(2)}\end{array}\right)$.

(24) If $i \in \operatorname{Seg} n$ and $\operatorname{rng} m_{1} \subseteq \operatorname{Seg}$ width $A$, then $\operatorname{Line}\left(\operatorname{Segm}\left(A, n_{1}, m_{1}\right), i\right)=$ $\operatorname{Line}\left(A, n_{1}(i)\right) \cdot m_{1}$. 
(25) If $i \in \operatorname{Seg} n$ and $j \in \operatorname{Seg} n$ and $n_{1}(i)=n_{1}(j)$, then $\operatorname{Line}\left(\operatorname{Segm}\left(A, n_{1}, m_{1}\right), i\right)=\operatorname{Line}\left(\operatorname{Segm}\left(A, n_{1}, m_{1}\right), j\right)$.

(26) If $i \in \operatorname{Seg} n$ and $j \in \operatorname{Seg} n$ and $n_{1}(i)=n_{1}(j)$ and $i \neq j$, then $\operatorname{Det} \operatorname{Segm}\left(M, n_{1}, n_{2}\right)=0_{K}$.

(27) If $n_{1}$ is not one-to-one, then $\operatorname{Det} \operatorname{Segm}\left(M, n_{1}, n_{2}\right)=0_{K}$.

(28) If $j \in \operatorname{Seg} m$ and $\operatorname{rng} n_{1} \subseteq \operatorname{Seg} \operatorname{len} A$, then $\left(\operatorname{Segm}\left(A, n_{1}, m_{1}\right)\right)_{\square, j}=$ $A_{\square, m_{1}(j)} \cdot n_{1}$.

(29) If $i \in \operatorname{Seg} m$ and $j \in \operatorname{Seg} m$ and $m_{1}(i)=m_{1}(j)$, then $\left(\operatorname{Segm}\left(A, n_{1}, m_{1}\right)\right)_{\square, i}=\left(\operatorname{Segm}\left(A, n_{1}, m_{1}\right)\right)_{\square, j}$.

(30) If $i \in \operatorname{Seg} m$ and $j \in \operatorname{Seg} m$ and $m_{1}(i)=m_{1}(j)$ and $i \neq j$, then $\operatorname{Det} \operatorname{Segm}\left(M, m_{2}, m_{1}\right)=0_{K}$.

(31) If $m_{1}$ is not one-to-one, then $\operatorname{Det} \operatorname{Segm}\left(M, m_{2}, m_{1}\right)=0_{K}$.

(32) Let $n_{1}, n_{2}$ be elements of $\mathbb{N}^{n}$. Suppose $n_{1}$ is one-to-one and $n_{2}$ is oneto-one and $\operatorname{rng} n_{1}=\operatorname{rng} n_{2}$. Then there exists a permutation $p_{1}$ of $\operatorname{Seg} n$ such that $n_{2}=n_{1} \cdot p_{1}$.

(33) For every function $f$ from $\operatorname{Seg} n$ into $\operatorname{Seg} n$ such that $n_{2}=n_{1} \cdot f$ holds $\operatorname{Segm}\left(A, n_{2}, m_{1}\right)=\operatorname{Segm}\left(A, n_{1}, m_{1}\right) \cdot f$.

(34) For every function $f$ from Seg $m$ into Seg $m$ such that $m_{2}=m_{1} \cdot f$ holds $\left(\operatorname{Segm}\left(A, n_{1}, m_{2}\right)\right)^{\mathrm{T}}=\left(\operatorname{Segm}\left(A, n_{1}, m_{1}\right)\right)^{\mathrm{T}} \cdot f$.

(35) Let $p_{1}$ be an element of the permutations of $n$-element set. If $n_{2}=$ $n_{3} \cdot p_{1}$, then $\operatorname{Det} \operatorname{Segm}\left(M, n_{2}, n_{1}\right)=(-1)^{\operatorname{sgn}\left(p_{1}\right)} \operatorname{Det} \operatorname{Segm}\left(M, n_{3}, n_{1}\right)$ and $\operatorname{Det} \operatorname{Segm}\left(M, n_{1}, n_{2}\right)=(-1)^{\operatorname{sgn}\left(p_{1}\right)} \operatorname{Det} \operatorname{Segm}\left(M, n_{1}, n_{3}\right)$.

(36) For all elements $n_{1}, n_{2}, n_{1}^{\prime}, n_{2}^{\prime}$ of $\mathbb{N}^{n}$ such that $\operatorname{rng} n_{1}=\operatorname{rng} n_{1}^{\prime}$ and $\operatorname{rng} n_{2}=\operatorname{rng} n_{2}^{\prime}$ holds $\operatorname{Det} \operatorname{Segm}\left(M, n_{1}, n_{2}\right)=\operatorname{Det} \operatorname{Segm}\left(M, n_{1}^{\prime}, n_{2}^{\prime}\right)$ or $\operatorname{Det} \operatorname{Segm}\left(M, n_{1}, n_{2}\right)=-\operatorname{Det} \operatorname{Segm}\left(M, n_{1}^{\prime}, n_{2}^{\prime}\right)$.

(37) Let $F, F_{1}$ be finite sequences of elements of $D$ and given $n_{1}, m_{1}$. Suppose len $F=$ width $A^{\prime}$ and $F_{1}=F \cdot m_{1}$ and $\left[\operatorname{rng} n_{1}, \operatorname{rng} m_{1}: \subseteq\right.$ the indices of $A^{\prime}$. Let given $i, j$. If $n_{1}^{-1}(\{j\})=\{i\}$, then $\mathrm{RLine}\left(\operatorname{Segm}\left(A^{\prime}, n_{1}, m_{1}\right), i, F_{1}\right)=$ $\operatorname{Segm}\left(\operatorname{RLine}\left(A^{\prime}, j, F\right), n_{1}, m_{1}\right)$.

(38) Let $F$ be a finite sequence of elements of $D$ and given $i, n_{1}$. If $i \notin$ $\operatorname{rng} n_{1}$ and $\left[\operatorname{rng} n_{1}, \operatorname{rng} m_{1}\right] \subseteq$ the indices of $A^{\prime}$, then $\operatorname{Segm}\left(A^{\prime}, n_{1}, m_{1}\right)=$ $\operatorname{Segm}\left(\operatorname{RLine}\left(A^{\prime}, i, F\right), n_{1}, m_{1}\right)$.

(39) If $i \in \operatorname{Seg} n^{\prime}$ and $i \in \operatorname{rng} n_{1}$ and $\left[\operatorname{rng} n_{1}, \operatorname{rng} m_{1}: \subseteq\right.$ the indices of $A^{\prime}$, then there exists $n_{2}$ such that $\operatorname{rng} n_{2}=\left(\operatorname{rng} n_{1} \backslash\{i\}\right) \cup\{j\}$ and $\operatorname{Segm}\left(\operatorname{RLine}\left(A^{\prime}, i, \operatorname{Line}\left(A^{\prime}, j\right)\right), n_{1}, m_{1}\right)=\operatorname{Segm}\left(A^{\prime}, n_{2}, m_{1}\right)$.

(40) For every finite sequence $F$ of elements of $D$ such that $i \notin \operatorname{Seg}$ len $A^{\prime}$ holds RLine $\left(A^{\prime}, i, F\right)=A^{\prime}$.

Let $n, m$ be natural numbers, let $K$ be a field, let $M$ be a matrix over $K$ of dimension $n \times m$, and let $a$ be an element of $K$. Then $a \cdot M$ is a matrix over 
$K$ of dimension $n \times m$.

We now state two propositions:

(41) If $\left[: \operatorname{rng} n_{1}, \operatorname{rng} m_{1}: \subseteq\right.$ the indices of $M$, then $a \cdot \operatorname{Segm}\left(M, n_{1}, m_{1}\right)=$ $\operatorname{Segm}\left(a \cdot M, n_{1}, m_{1}\right)$.

(42) If $n_{1}=\operatorname{idseq}(\operatorname{len} A)$ and $m_{1}=\operatorname{idseq}($ width $A)$, then $\operatorname{Segm}\left(A, n_{1}, m_{1}\right)=$ $A$.

Let us observe that there exists a subset of $\mathbb{N}$ which is empty, without zero, and finite and there exists a subset of $\mathbb{N}$ which is non empty, without zero, and finite.

Let us consider $n$. Observe that $\operatorname{Seg} n$ is without zero.

Let $X$ be a without zero set and let $Y$ be a set. One can verify that $X \backslash Y$ is without zero and $X \cap Y$ is without zero.

One can prove the following proposition

(43) For every finite without zero subset $N$ of $\mathbb{N}$ there exists $k$ such that $N \subseteq \operatorname{Seg} k$.

Let $N$ be a finite without zero subset of $\mathbb{N}$. Then $\operatorname{Sgm} N$ is an element of $\mathbb{N}^{\operatorname{card} N}$.

Let $D$ be a non empty set, let $A$ be a matrix over $D$, and let $P, Q$ be without zero finite subsets of $\mathbb{N}$. The functor $\operatorname{Segm}(A, P, Q)$ yields a matrix over $D$ of dimension card $P \times \operatorname{card} Q$ and is defined by:

(Def. 2) $\operatorname{Segm}(A, P, Q)=\operatorname{Segm}(A, \operatorname{Sgm} P, \operatorname{Sgm} Q)$.

Next we state two propositions:

(44) $\operatorname{Segm}\left(A,\left\{i_{0}\right\},\left\{j_{0}\right\}\right)=\left\langle\left\langle A_{i_{0}, j_{0}}\right\rangle\right\rangle$.

(45) If $i_{0}<j_{0}$ and $n_{0}<m_{0}$, then $\operatorname{Segm}\left(A,\left\{i_{0}, j_{0}\right\},\left\{n_{0}, m_{0}\right\}\right)=$ $\left(\begin{array}{cc}A_{i_{0}, n_{0}} & A_{i_{0}, m_{0}} \\ A_{j_{0}, n_{0}} & A_{j_{0}, m_{0}}\end{array}\right)$.

In the sequel $P, P_{1}, P_{2}, Q, Q_{1}, Q_{2}$ are without zero finite subsets of $\mathbb{N}$.

The following propositions are true:

(46) $\operatorname{Segm}(A, \operatorname{Seg}$ len $A, \operatorname{Seg}$ width $A)=A$.

(47) If $i \in \operatorname{Seg} \operatorname{card} P$ and $Q \subseteq \operatorname{Seg}$ width $A$, then $\operatorname{Line}(\operatorname{Segm}(A, P, Q), i)=$ $\operatorname{Line}(A,(\operatorname{Sgm} P)(i)) \cdot \operatorname{Sgm} Q$.

(48) If $i \in \operatorname{Seg} \operatorname{card} P$, then $\operatorname{Line}(\operatorname{Segm}(A, P, \operatorname{Seg}$ width $A), i)=$ Line $(A,(\operatorname{Sgm} P)(i))$.

(49) If $j \in \operatorname{Seg} \operatorname{card} Q$ and $P \subseteq \operatorname{Seg} \operatorname{len} A$, then $(\operatorname{Segm}(A, P, Q))_{\square, j}=$ $A_{\square,(\operatorname{Sgm} Q)(j)} \cdot \operatorname{Sgm} P$.

(50) If $j \in \operatorname{Seg} \operatorname{card} Q$, then $(\operatorname{Segm}(A, \operatorname{Seg} \operatorname{len} A, Q))_{\square, j}=A_{\square,(\operatorname{Sgm} Q)(j)}$.

(51) $\operatorname{Segm}(A, \operatorname{Seg}$ len $A \backslash\{i\}, \operatorname{Seg}$ width $A)=A_{\lceil i}$.

(52) $\operatorname{Segm}(M$, Seg len $M$, Seg width $M \backslash\{i\})=$ the deleting of $i$-column in $M$.

(53) $(\operatorname{Sgm} P)^{-1}(X)$ is a without zero finite subset of $\mathbb{N}$. 
(54) If $X \subseteq P$, then $\operatorname{Sgm} X=\operatorname{Sgm} P \cdot \operatorname{Sgm}\left((\operatorname{Sgm} P)^{-1}(X)\right)$.

(55) $\quad\left[(\operatorname{Sgm} P)^{-1}(X),(\operatorname{Sgm} Q)^{-1}(Y): \subseteq\right.$ the indices of $\operatorname{Segm}(A, P, Q)$.

(56) If $P \subseteq P_{1}$ and $Q \subseteq Q_{1}$ and $P_{2}=\left(\operatorname{Sgm} P_{1}\right)^{-1}(P)$ and $Q_{2}=\left(\operatorname{Sgm} Q_{1}\right)^{-1}(Q)$, then $\left[\operatorname{rng} \operatorname{Sgm} P_{2}, \operatorname{rng} \operatorname{Sgm} Q_{2}: \subseteq\right.$ the indices of $\operatorname{Segm}\left(A, P_{1}, Q_{1}\right)$ and $\operatorname{Segm}\left(\operatorname{Segm}\left(A, P_{1}, Q_{1}\right), P_{2}, Q_{2}\right)=\operatorname{Segm}(A, P, Q)$.

(57) Suppose $P=\emptyset$ iff $Q=\emptyset$ and $: P, Q: \subseteq$ the indices of $\operatorname{Segm}\left(A, P_{1}, Q_{1}\right)$. Then there exist $P_{2}, Q_{2}$ such that $P_{2} \subseteq P_{1}$ and $Q_{2} \subseteq Q_{1}$ and $P_{2}=$ $\left(\operatorname{Sgm} P_{1}\right)^{\circ} P$ and $Q_{2}=\left(\operatorname{Sgm} Q_{1}\right)^{\circ} Q$ and $\operatorname{card} P_{2}=\operatorname{card} P$ and $\operatorname{card} Q_{2}=$ $\operatorname{card} Q$ and $\operatorname{Segm}\left(\operatorname{Segm}\left(A, P_{1}, Q_{1}\right), P, Q\right)=\operatorname{Segm}\left(A, P_{2}, Q_{2}\right)$.

(58) For every matrix $M$ over $K$ of dimension $n$ holds $\operatorname{Segm}(M, \operatorname{Seg} n \backslash$ $\{i\}, \operatorname{Seg} n \backslash\{j\})=$ the deleting of $i$-row and $j$-column in $M$.

(59) Let $F, F_{2}$ be finite sequences of elements of $D$. Suppose len $F=$ width $A^{\prime}$ and $F_{2}=F \cdot \operatorname{Sgm} Q$ and $: P, Q: \subseteq$ the indices of $A^{\prime}$. Then $\operatorname{RLine}\left(\operatorname{Segm}\left(A^{\prime}, P, Q\right), i, F_{2}\right)=\operatorname{Segm}\left(\operatorname{RLine}\left(A^{\prime},(\operatorname{Sgm} P)(i), F\right), P, Q\right)$.

(60) Let $F$ be a finite sequence of elements of $D$ and given $i, P$. If $i \notin P$ and $\left[P, Q: \subseteq\right.$ the indices of $A^{\prime}$, then $\operatorname{Segm}\left(A^{\prime}, P, Q\right)=$ $\operatorname{Segm}\left(\operatorname{RLine}\left(A^{\prime}, i, F\right), P, Q\right)$.

(61) If $[: P, Q: \subseteq$ the indices of $A$ and $\operatorname{card} P=0$ iff $\operatorname{card} Q=0$, then $(\operatorname{Segm}(A, P, Q))^{\mathrm{T}}=\operatorname{Segm}\left(A^{\mathrm{T}}, Q, P\right)$.

(62) If : $P, Q: \subseteq$ the indices of $A$ and if $\operatorname{card} Q=0$, then $\operatorname{card} P=0$, then $\operatorname{Segm}(A, P, Q)=\left(\operatorname{Segm}\left(A^{\mathrm{T}}, Q, P\right)\right)^{\mathrm{T}}$.

(63) If $[: P, Q: \subseteq$ the indices of $M$, then $a \cdot \operatorname{Segm}(M, P, Q)=\operatorname{Segm}(a \cdot M, P, Q)$.

Let $D$ be a non empty set, let $A$ be a matrix over $D$, and let $P, Q$ be without zero finite subsets of $\mathbb{N}$. Let us assume that card $P=\operatorname{card} Q$. The functor $\operatorname{EqSegm}(A, P, Q)$ yields a matrix over $D$ of dimension card $P$ and is defined by:

(Def. 3) $\operatorname{EqSegm}(A, P, Q)=\operatorname{Segm}(A, P, Q)$.

Next we state several propositions:

(64) For all $P, Q, i, j$ such that $i \in \operatorname{Seg} \operatorname{card} P$ and $j \in \operatorname{Seg} \operatorname{card} P$ and card $P=\operatorname{card} Q$ holds Delete $(\operatorname{EqSegm}(M, P, Q), i, j)=\operatorname{EqSegm}(M, P \backslash$ $\{(\operatorname{Sgm} P)(i)\}, Q \backslash\{(\operatorname{Sgm} Q)(j)\})$ and $\operatorname{card}(P \backslash\{(\operatorname{Sgm} P)(i)\})=\operatorname{card}(Q \backslash$ $\{(\operatorname{Sgm} Q)(j)\})$.

(65) For all $M, P, P_{1}, Q_{1}$ such that card $P_{1}=\operatorname{card} Q_{1}$ and $P \subseteq P_{1}$ and $\operatorname{Det} \operatorname{EqSegm}\left(M, P_{1}, Q_{1}\right) \neq 0_{K}$ there exists $Q$ such that $Q \subseteq Q_{1}$ and card $P=\operatorname{card} Q$ and $\operatorname{Det} \operatorname{EqSegm}(M, P, Q) \neq 0_{K}$.

(66) For all $M, P_{1}, Q, Q_{1}$ such that card $P_{1}=\operatorname{card} Q_{1}$ and $Q \subseteq Q_{1}$ and $\operatorname{Det} \operatorname{EqSegm}\left(M, P_{1}, Q_{1}\right) \neq 0_{K}$ there exists $P$ such that $P \subseteq P_{1}$ and card $P=\operatorname{card} Q$ and $\operatorname{Det} \operatorname{EqSegm}(M, P, Q) \neq 0_{K}$.

(67) If $\operatorname{card} P=\operatorname{card} Q$, then $[P, Q] \subseteq$ the indices of $A$ iff $P \subseteq \operatorname{Seg} \operatorname{len} A$ 
and $Q \subseteq \operatorname{Seg}$ width $A$.

(68) Let given $P, Q, i, j_{0}$. Suppose $i \in \operatorname{Seg} n^{\prime}$ and $j_{0} \in \operatorname{Seg} n^{\prime}$ and $i \in P$ and $j_{0} \notin P$ and $\operatorname{card} P=\operatorname{card} Q$ and $: P, Q: \subseteq$ the indices of $M^{\prime}$. Then card $P=\operatorname{card}\left((P \backslash\{i\}) \cup\left\{j_{0}\right\}\right)$ but $:(P \backslash\{i\}) \cup\left\{j_{0}\right\}, Q: \subseteq$ the indices of $M^{\prime}$ but Det EqSegm $\left(\operatorname{RLine}\left(M^{\prime}, i, \operatorname{Line}\left(M^{\prime}, j_{0}\right)\right), P, Q\right)=\operatorname{Det} \operatorname{EqSegm}\left(M^{\prime},(P \backslash\right.$ $\left.\{i\}) \cup\left\{j_{0}\right\}, Q\right)$ or $\operatorname{Det} \operatorname{EqSegm}\left(\operatorname{RLine}\left(M^{\prime}, i, \operatorname{Line}\left(M^{\prime}, j_{0}\right)\right), P, Q\right)=$ $-\operatorname{Det} \operatorname{EqSegm}\left(M^{\prime},(P \backslash\{i\}) \cup\left\{j_{0}\right\}, Q\right)$.

(69) If $\operatorname{card} P=\operatorname{card} Q$, then $[: P, Q] \subseteq$ the indices of $A$ iff $[: Q, P: \subseteq$ the indices of $A^{\mathrm{T}}$.

(70) If $[P, Q: \subseteq$ the indices of $M$ and $\operatorname{card} P=\operatorname{card} Q$, then $\operatorname{Det} \operatorname{EqSegm}(M, P, Q)=\operatorname{Det} \operatorname{EqSegm}\left(M^{\mathrm{T}}, Q, P\right)$.

(71) For every matrix $M$ over $K$ of dimension $n$ holds $\operatorname{Det}(a \cdot M)=\operatorname{power}_{K}(a$, $n) \cdot \operatorname{Det} M$.

(72) If $[: P, Q: \subseteq$ the indices of $M$ and $\operatorname{card} P=\operatorname{card} Q$, then $\operatorname{Det} \operatorname{EqSegm}(a$. $M, P, Q)=\operatorname{power}_{K}(a, \operatorname{card} P) \cdot \operatorname{Det} \operatorname{EqSegm}(M, P, Q)$.

Let $K$ be a field and let $M$ be a matrix over $K$. The functor $\operatorname{rk}(M)$ yielding an element of $\mathbb{N}$ is defined by the conditions (Def. 4).

(Def. 4)(i) There exist $P, Q$ such that $: P, Q: \subseteq$ the indices of $M$ and $\operatorname{card} P=$ card $Q$ and $\operatorname{card} P=\operatorname{rk}(M)$ and $\operatorname{Det} \operatorname{EqSegm}(M, P, Q) \neq 0_{K}$, and

(ii) for all $P_{1}, Q_{1}$ such that $: P_{1}, Q_{1}: \subseteq$ the indices of $M$ and card $P_{1}=$ card $Q_{1}$ and $\operatorname{Det} \operatorname{EqSegm}\left(M, P_{1}, Q_{1}\right) \neq 0_{K}$ holds card $P_{1} \leq \operatorname{rk}(M)$.

The following propositions are true:

(73) For all $P, Q$ such that $[P, Q: \subseteq$ the indices of $M$ and $\operatorname{card} P=\operatorname{card} Q$ holds card $P \leq$ len $M$ and $\operatorname{card} Q \leq$ width $M$.

(74) $\operatorname{rk}(M) \leq \operatorname{len} M$ and $\operatorname{rk}(M) \leq$ width $M$.

(75) If $\left[: \operatorname{rng} n_{2}, \operatorname{rng} n_{3}: \subseteq\right.$ the indices of $M$ and $\operatorname{Det} \operatorname{Segm}\left(M, n_{2}, n_{3}\right) \neq 0_{K}$, then there exist $P_{1}, P_{2}$ such that $P_{1}=\operatorname{rng} n_{2}$ and $P_{2}=\operatorname{rng} n_{3}$ and card $P_{1}=\operatorname{card} P_{2}$ and $\operatorname{card} P_{1}=n$ and $\operatorname{Det} \operatorname{EqSegm}\left(M, P_{1}, P_{2}\right) \neq 0_{K}$.

(76) Let $R_{1}$ be an element of $\mathbb{N}$. Then $\operatorname{rk}(M)=R_{1}$ if and only if the following conditions are satisfied:

(i) there exist elements $r_{1}, r_{2}$ of $\mathbb{N}^{R_{1}}$ such that $\left[\operatorname{rng} r_{1}, \operatorname{rng} r_{2}: \subseteq\right.$ the indices of $M$ and $\operatorname{Det} \operatorname{Segm}\left(M, r_{1}, r_{2}\right) \neq 0_{K}$, and

(ii) for all $n, n_{2}, n_{3}$ such that $\left[\operatorname{rng} n_{2}, \operatorname{rng} n_{3}: \subseteq\right.$ the indices of $M$ and $\operatorname{Det} \operatorname{Segm}\left(M, n_{2}, n_{3}\right) \neq 0_{K}$ holds $n \leq R_{1}$.

(77) If $n=0$ or $m=0$, then $\operatorname{rk}\left(\operatorname{Segm}\left(M, n_{1}, m_{1}\right)\right)=0$.

(78) If $\left[\operatorname{rng} n_{1}, \operatorname{rng} m_{1}\right] \subseteq \subseteq$ the indices of $M$, then $\operatorname{rk}(M) \quad \geq$ $\operatorname{rk}\left(\operatorname{Segm}\left(M, n_{1}, m_{1}\right)\right)$.

(79) If $[: P, Q: \subseteq$ the indices of $M$, then $\operatorname{rk}(M) \geq \operatorname{rk}(\operatorname{Segm}(M, P, Q))$.

(80) If $P \subseteq P_{1}$ and $Q \subseteq Q_{1}$, then $\operatorname{rk}(\operatorname{Segm}(M, P, Q)) \leq \operatorname{rk}\left(\operatorname{Segm}\left(M, P_{1}, Q_{1}\right)\right)$. 
(81) For all functions $f, g$ such that $\operatorname{rng} f \subseteq \operatorname{rng} g$ there exists a function $h$ such that $\operatorname{dom} h=\operatorname{dom} f$ and $\operatorname{rng} h \subseteq \operatorname{dom} g$ and $f=g \cdot h$.

(82) If $\left[\operatorname{rng} n_{1}, \operatorname{rng} m_{1}:=\right.$ the indices of $M$, then $\operatorname{rk}(M)=$ $\operatorname{rk}\left(\operatorname{Segm}\left(M, n_{1}, m_{1}\right)\right)$.

(83) For every matrix $M$ over $K$ of dimension $n$ holds $\operatorname{rk}(M)=n$ iff $\operatorname{Det} M \neq$ $0_{K}$.

(84) $\operatorname{rk}(M)=\operatorname{rk}\left(M^{\mathrm{T}}\right)$.

(85) For every matrix $M$ over $K$ of dimension $n \times m$ and for every permutation $F$ of $\operatorname{Seg} n$ holds $\operatorname{rk}(M)=\operatorname{rk}(M \cdot F)$.

(86) If $a \neq 0_{K}$, then $\operatorname{rk}(M)=\operatorname{rk}(a \cdot M)$.

(87) Let $p, p_{2}$ be finite sequences of elements of $K$ and $f$ be a function. If $p_{2}=p \cdot f$ and $\operatorname{rng} f \subseteq \operatorname{dom} p$, then $a \cdot p \cdot f=a \cdot p_{2}$.

(88) Let $p, p_{2}, q, q_{1}$ be finite sequences of elements of $K$ and $f$ be a function. If $p_{2}=p \cdot f$ and $\operatorname{rng} f \subseteq \operatorname{dom} p$ and $q_{1}=q \cdot f$ and $\operatorname{rng} f \subseteq \operatorname{dom} q$, then $(p+q) \cdot f=p_{2}+q_{1}$.

(89) If $a \neq 0_{K}$, then $\operatorname{rk}\left(M^{\prime}\right)=\operatorname{rk}\left(\operatorname{RLine}\left(M^{\prime}, i, a \cdot \operatorname{Line}\left(M^{\prime}, i\right)\right)\right)$.

(90) If Line $(M, i)=$ width $M \mapsto 0_{K}$, then $\operatorname{rk}($ the deleting of $i$-row in $M)=$ $\operatorname{rk}(M)$.

(91) For every $p$ such that len $p=$ width $M^{\prime}$ holds rk(the deleting of $i$-row in $\left.M^{\prime}\right)=\operatorname{rk}\left(\operatorname{RLine}\left(M^{\prime}, i, 0_{K} \cdot p\right)\right)$.

(92) If $j \in$ Seglen $M^{\prime}$ and if $i=j$, then $a \neq-\mathbf{1}_{K}$, then $\operatorname{rk}\left(M^{\prime}\right)=$ $\operatorname{rk}\left(\operatorname{RLine}\left(M^{\prime}, i, \operatorname{Line}\left(M^{\prime}, i\right)+a \cdot \operatorname{Line}\left(M^{\prime}, j\right)\right)\right)$.

(93) If $j \in \operatorname{Seg}$ len $M^{\prime}$ and $j \neq i$, then $\operatorname{rk}\left(\right.$ the deleting of $i$-row in $\left.M^{\prime}\right)=$ $\operatorname{rk}\left(\operatorname{RLine}\left(M^{\prime}, i, a \cdot \operatorname{Line}\left(M^{\prime}, j\right)\right)\right)$.

(94) $\operatorname{rk}(M)>0$ iff there exist $i, j$ such that $\langle i, j\rangle \in$ the indices of $M$ and $M_{i, j} \neq 0_{K}$.

$\operatorname{rk}(M)=0$ iff $M=\left(\begin{array}{ccc}0 & \ldots & 0 \\ \vdots & \ddots & \vdots \\ 0 & \ldots & 0\end{array}\right)_{K}^{(\operatorname{len} M) \times(\operatorname{width} M)}$

(96) $\operatorname{rk}(M)=1$ if and only if the following conditions are satisfied:

(i) there exist $i, j$ such that $\langle i, j\rangle \in$ the indices of $M$ and $M_{i, j} \neq 0_{K}$, and

(ii) for all $i_{0}, j_{0}, n_{0}, m_{0}$ such that $i_{0} \neq j_{0}$ and $n_{0} \neq m_{0}$ and $:\left\{i_{0}, j_{0}\right\}$, $\left\{n_{0}, m_{0}\right\}: \subseteq$ the indices of $M$ holds $\operatorname{Det} \operatorname{EqSegm}\left(M,\left\{i_{0}, j_{0}\right\},\left\{n_{0}, m_{0}\right\}\right)=$ $0_{K}$.

(97) $\operatorname{rk}(M)=1$ if and only if the following conditions are satisfied:

(i) there exist $i, j$ such that $\langle i, j\rangle \in$ the indices of $M$ and $M_{i, j} \neq 0_{K}$, and

(ii) for all $i, j, n, m$ such that $:\{i, j\},\{n, m\}: \subseteq$ the indices of $M$ holds $M_{i, n} \cdot M_{j, m}=M_{i, m} \cdot M_{j, n}$. 
(98) $\operatorname{rk}(M)=1$ if and only if there exists $i$ such that $i \in \operatorname{Seg}$ len $M$ and there exists $j$ such that $j \in \operatorname{Seg}$ width $M$ and $M_{i, j} \neq 0_{K}$ and for every $k$ such that $k \in \operatorname{Seg}$ len $M$ there exists $a$ such that Line $(M, k)=a \cdot \operatorname{Line}(M, i)$.

Let us consider $K$. Observe that there exists a matrix over $K$ which is diagonal.

One can prove the following propositions:

(99) Let $M$ be a diagonal matrix over $K$ and $N_{1}$ be a set. Suppose $N_{1}=\{i$ : $\langle i, i\rangle \in$ the indices of $\left.M \wedge M_{i, i} \neq 0_{K}\right\}$. Let given $P$, $Q$. If : $P, Q: \subseteq$ the indices of $M$ and card $P=\operatorname{card} Q$ and $\operatorname{Det} \operatorname{EqSegm}(M, P, Q) \neq 0_{K}$, then $P \subseteq N_{1}$ and $Q \subseteq N_{1}$.

(100) For every diagonal matrix $M$ over $K$ and for every $P$ such that $: P$, $P: \subseteq$ the indices of $M$ holds $\operatorname{Segm}(M, P, P)$ is diagonal.

(101) Let $M$ be a diagonal matrix over $K$ and $N_{1}$ be a set. If $N_{1}=\{i:\langle i$, $i\rangle \in$ the indices of $\left.M \wedge M_{i, i} \neq 0_{K}\right\}$, then $\operatorname{rk}(M)=\overline{\overline{N_{1}}}$.

For simplicity, we adopt the following rules: $v, v_{1}, v_{2}, u$ denote vectors of the $n$-dimension vector space over $K, t, t_{1}, t_{2}$ denote elements of (the carrier of $K)^{n}, L$ denotes a linear combination of the $n$-dimension vector space over $K$, and $M, M_{1}$ denote matrices over $K$ of dimension $m \times n$.

We now state the proposition

(102)(i) The carrier of the $n$-dimension vector space over $K=$ (the carrier of $K)^{n}$,

(ii) $0_{\text {the }} n$-dimension vector space over $K=n \mapsto 0_{K}$,

(iii) if $t_{1}=v_{1}$ and $t_{2}=v_{2}$, then $t_{1}+t_{2}=v_{1}+v_{2}$, and

(iv) if $t=v$, then $a \cdot t=a \cdot v$.

Let us consider $K, n$. Then the $n$-dimension vector space over $K$ is a strict vector space over $K$.

Let us consider $K, n$. One can verify that every vector of the $n$-dimension vector space over $K$ is function-like and relation-like.

Let us consider $K, m, n$ and let $M$ be a matrix over $K$ of dimension $m \times$ $n$. We introduce lines $(M)$ as a synonym of $\operatorname{rng} M$. We introduce $M$ is without repeated line as a synonym of $M$ is one-to-one.

Let $K$ be a field, let us consider $m, n$, and let $M$ be a matrix over $K$ of dimension $m \times n$. Then $\operatorname{lines}(M)$ is a subset of the $n$-dimension vector space over $K$.

Next we state two propositions:

(103) $x \in \operatorname{lines}(M)$ iff there exists $i$ such that $i \in \operatorname{Seg} m$ and $x=\operatorname{Line}(M, i)$.

(104) Let $V$ be a finite subset of the $n$-dimension vector space over $K$. Then there exists a matrix $M$ over $K$ of dimension card $V \times n$ such that $M$ is without repeated line and lines $(M)=V$. 
Let us consider $K, n$ and let $F$ be a finite sequence of elements of the $n$ dimension vector space over $K$. The functor FinS2MX $F$ yielding a matrix over $K$ of dimension len $F \times n$ is defined by:

(Def. 5) FinS2MX $F=F$.

Let us consider $K, m, n$ and let $M$ be a matrix over $K$ of dimension $m$ $\times n$. The functor MX2FinS $M$ yielding a finite sequence of elements of the $n$-dimension vector space over $K$ is defined as follows:

(Def. 6) MX2FinS $M=M$.

One can prove the following propositions:

(105) If $\operatorname{rk}(M)=m$, then $M$ is without repeated line.

(106) If $i \in \operatorname{Seg} \operatorname{len} M$ and $a=L(M(i))$, then Line $(\operatorname{FinS} 2 \mathrm{MX}(L \mathrm{MX} 2 \mathrm{FinS} M), i)=a \cdot \operatorname{Line}(M, i)$.

(107) If $M$ is without repeated line and the support of $L \subseteq \operatorname{lines}(M)$ and $i \in \operatorname{Seg} n$, then $\left(\sum L\right)(i)=\sum\left((\operatorname{FinS} 2 \mathrm{MX}(L \operatorname{MX} 2 \operatorname{FinS} M))_{\square, i}\right)$.

(108) Let given $M, M_{1}$. Suppose $M$ is without repeated line and for every $i$ such that $i \in \operatorname{Seg} m$ there exists $a$ such that $\operatorname{Line}\left(M_{1}, i\right)=$ $a \cdot \operatorname{Line}(M, i)$. Then there exists a linear combination $L$ of lines $(M)$ such that $L$ MX2FinS $M=M_{1}$.

(109) Let given $M$. Suppose $M$ is without repeated line. Then for every $i$ such that $i \in \operatorname{Seg} m$ holds $\operatorname{Line}(M, i) \neq n \mapsto 0_{K}$ and for every $M_{1}$ such that for every $i$ such that $i \in \operatorname{Seg} m$ there exists $a$ such that $\operatorname{Line}\left(M_{1}, i\right)=$ $a \cdot \operatorname{Line}(M, i)$ and for every $j$ such that $j \in \operatorname{Seg} n$ holds $\sum\left(\left(M_{1}\right) \square, j\right)=$ $0_{K}$ holds $M_{1}=\left(\begin{array}{ccc}0 & \ldots & 0 \\ \vdots & \ddots & \vdots \\ 0 & \ldots & 0\end{array}\right)_{K}^{m \times n}$ if and only if lines $(M)$ is linearly independent.

(110) If $\operatorname{rk}(M)=m$, then lines $(M)$ is linearly independent.

(111) Let $M$ be a diagonal $n$-dimensional matrix over $K$. Suppose $\operatorname{rk}(M)=n$. Then lines $(M)$ is a basis of the $n$-dimension vector space over $K$.

Let us consider $K, n$. Then the $n$-dimension vector space over $K$ is a strict finite dimensional vector space over $K$.

The following propositions are true:

(112) $\operatorname{dim}($ the $n$-dimension vector space over $K)=n$.

(113) Let given $M, i, a$. Suppose that for every $j$ such that $j \in \operatorname{Seg} m$ holds $M_{j, i}=a$. Then $M$ is without repeated line if and only if $\operatorname{Segm}(M, \operatorname{Seg}$ len $M, \operatorname{Seg}$ width $M \backslash\{i\})$ is without repeated line.

(114) Let given $M, i$. Suppose $M$ is without repeated line and $\operatorname{lines}(M)$ is linearly independent and for every $j$ such that $j \in \operatorname{Seg} m$ holds $M_{j, i}=0_{K}$. Then lines $(\operatorname{Segm}(M$, Seg len $M$, Seg width $M \backslash\{i\}))$ is linearly independent. 
(115) Let $V$ be a vector space over $K$ and $U$ be a finite subset of $V$. Suppose $U$ is linearly independent. Let $u, v$ be vectors of $V$. If $u \in U$ and $v \in U$ and $u \neq v$, then $(U \backslash\{u\}) \cup\{u+a \cdot v\}$ is linearly independent.

(116) Let $V$ be a vector space over $K$ and $u, v$ be vectors of $V$. Then $x \in$ $\operatorname{Lin}(\{u, v\})$ if and only if there exist $a, b$ such that $x=a \cdot u+b \cdot v$.

(117) Let given $M$. Suppose lines $(M)$ is linearly independent and $M$ is without repeated line. Let given $i, j$. Suppose $j \in \operatorname{Seg} \operatorname{len} M$ and $i \neq j$. Then $\operatorname{RLine}(M, i$, Line $(M, i)+a \cdot \operatorname{Line}(M, j))$ is without repeated line and lines $(\operatorname{RLine}(M, i, \operatorname{Line}(M, i)+a \cdot \operatorname{Line}(M, j)))$ is linearly independent.

(118) If $P \subseteq \operatorname{Seg} m$, then lines $(\operatorname{Segm}(M, P, \operatorname{Seg} n)) \subseteq \operatorname{lines}(M)$.

(119) If $P \subseteq \operatorname{Seg} m$ and lines $(M)$ is linearly independent, then lines $(\operatorname{Segm}(M, P, \operatorname{Seg} n))$ is linearly independent.

(120) If $P \subseteq \operatorname{Seg} m$ and $M$ is without repeated line, then $\operatorname{Segm}(M, P, \operatorname{Seg} n)$ is without repeated line.

(121) Let $M$ be a matrix over $K$ of dimension $m \times n$. Then lines $(M)$ is linearly independent and $M$ is without repeated line if and only if $\operatorname{rk}(M)=m$.

(122) Let $U$ be a subset of the $n$-dimension vector space over $K$. Suppose $U \subseteq \operatorname{lines}(M)$. Then there exists $P$ such that $P \subseteq \operatorname{Seg} m$ and $\operatorname{lines}(\operatorname{Segm}(M, P, \operatorname{Seg} n))=U$ and $\operatorname{Segm}(M, P, \operatorname{Seg} n)$ is without repeated line.

(123) Let $R_{1}$ be an element of $\mathbb{N}$. Then $\operatorname{rk}(M)=R_{1}$ if and only if the following conditions are satisfied:

(i) there exists a finite subset $U$ of the $n$-dimension vector space over $K$ such that $U$ is linearly independent and $U \subseteq \operatorname{lines}(M)$ and card $U=R_{1}$, and

(ii) for every finite subset $W$ of the $n$-dimension vector space over $K$ such that $W$ is linearly independent and $W \subseteq \operatorname{lines}(M)$ holds card $W \leq R_{1}$.

\section{REFERENCES}

[1] Grzegorz Bancerek. Cardinal numbers. Formalized Mathematics, 1(2):377-382, 1990.

[2] Grzegorz Bancerek. The fundamental properties of natural numbers. Formalized Mathematics, 1(1):41-46, 1990.

[3] Grzegorz Bancerek and Krzysztof Hryniewiecki. Segments of natural numbers and finite sequences. Formalized Mathematics, 1(1):107-114, 1990.

[4] Czesław Byliński. Binary operations. Formalized Mathematics, 1(1):175-180, 1990.

[5] Czesław Byliński. Binary operations applied to finite sequences. Formalized Mathematics, 1(4):643-649, 1990.

[6] Czesław Byliński. Finite sequences and tuples of elements of a non-empty sets. Formalized Mathematics, 1(3):529-536, 1990.

[7] Czesław Byliński. Functions and their basic properties. Formalized Mathematics, 1(1):5565, 1990.

[8] Czesław Byliński. Functions from a set to a set. Formalized Mathematics, 1(1):153-164, 1990.

[9] Czesław Byliński. The modification of a function by a function and the iteration of the composition of a function. Formalized Mathematics, 1(3):521-527, 1990. 
[10] Czesław Byliński. Some basic properties of sets. Formalized Mathematics, 1(1):47-53, 1990.

[11] Czesław Byliński. Some properties of restrictions of finite sequences. Formalized Mathematics, 5(2):241-245, 1996.

[12] Agata Darmochwał. Finite sets. Formalized Mathematics, 1(1):165-167, 1990.

[13] Katarzyna Jankowska. Matrices. Abelian group of matrices. Formalized Mathematics, 2(4):475-480, 1991.

[14] Katarzyna Jankowska. Transpose matrices and groups of permutations. Formalized Mathematics, 2(5):711-717, 1991.

[15] Eugeniusz Kusak, Wojciech Leończuk, and Michał Muzalewski. Abelian groups, fields and vector spaces. Formalized Mathematics, 1(2):335-342, 1990.

[16] Rafał Kwiatek. Factorial and Newton coefficients. Formalized Mathematics, 1(5):887-890, 1990.

[17] Anna Lango and Grzegorz Bancerek. Product of families of groups and vector spaces. Formalized Mathematics, 3(2):235-240, 1992.

[18] Yatsuka Nakamura. Determinant of some matrices of field elements. Formalized Mathematics, 14(1):1-5, 2006.

[19] Takaya Nishiyama and Yasuho Mizuhara. Binary arithmetics. Formalized Mathematics, 4(1):83-86, 1993.

[20] Karol Pąk. Basic properties of determinants of square matrices over a field. Formalized Mathematics, 15(1):17-25, 2007.

[21] Karol Pa̧k and Andrzej Trybulec. Laplace expansion. Formalized Mathematics, 15(3):143$150,2007$.

[22] Yozo Toda. The formalization of simple graphs. Formalized Mathematics, 5(1):137-144, 1996.

[23] Andrzej Trybulec. Subsets of complex numbers. To appear in Formalized Mathematics.

[24] Andrzej Trybulec. Binary operations applied to functions. Formalized Mathematics, 1(2):329-334, 1990.

[25] Andrzej Trybulec. Domains and their Cartesian products. Formalized Mathematics, 1(1):115-122, 1990.

[26] Andrzej Trybulec. Function domains and Frænkel operator. Formalized Mathematics, 1(3):495-500, 1990.

[27] Andrzej Trybulec. Tarski Grothendieck set theory. Formalized Mathematics, 1(1):9-11, 1990.

[28] Andrzej Trybulec and Agata Darmochwał. Boolean domains. Formalized Mathematics, 1(1):187-190, 1990.

[29] Wojciech A. Trybulec. Basis of vector space. Formalized Mathematics, 1(5):883-885, 1990.

[30] Wojciech A. Trybulec. Binary operations on finite sequences. Formalized Mathematics, 1(5):979-981, 1990.

[31] Wojciech A. Trybulec. Groups. Formalized Mathematics, 1(5):821-827, 1990.

[32] Wojciech A. Trybulec. Linear combinations in vector space. Formalized Mathematics, $1(5): 877-882,1990$.

[33] Wojciech A. Trybulec. Non-contiguous substrings and one-to-one finite sequences. Formalized Mathematics, 1(3):569-573, 1990.

[34] Wojciech A. Trybulec. Pigeon hole principle. Formalized Mathematics, 1(3):575-579, 1990.

[35] Wojciech A. Trybulec. Subspaces and cosets of subspaces in vector space. Formalized Mathematics, 1(5):865-870, 1990.

[36] Wojciech A. Trybulec. Vectors in real linear space. Formalized Mathematics, 1(2):291296, 1990.

[37] Zinaida Trybulec. Properties of subsets. Formalized Mathematics, 1(1):67-71, 1990.

[38] Edmund Woronowicz. Relations and their basic properties. Formalized Mathematics, 1(1):73-83, 1990.

[39] Edmund Woronowicz. Relations defined on sets. Formalized Mathematics, 1(1):181-186, 1990.

[40] Xiaopeng Yue, Xiquan Liang, and Zhongpin Sun. Some properties of some special matrices. Formalized Mathematics, 13(4):541-547, 2005.

[41] Katarzyna Zawadzka. The sum and product of finite sequences of elements of a field. Formalized Mathematics, 3(2):205-211, 1992. 
[42] Katarzyna Zawadzka. The product and the determinant of matrices with entries in a field. Formalized Mathematics, 4(1):1-8, 1993.

[43] Mariusz Żynel. The Steinitz theorem and the dimension of a vector space. Formalized Mathematics, 5(3):423-428, 1996.

Received August 28, 2007 